\section{Übelkeit und Erbrechen oft unterschätzt}

\section{Für Krebspatienten gehören Übelkeit und Erbrechen zu den belastendsten Nebenwirkungen von Chemo- und Strahlentherapie. Doch nehmen Ärzte und Pflegepersonal die Problematik genauso wahr wie ihre Patienten?}

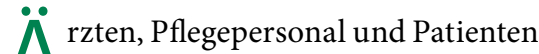

in 5 europäischen Ländern (375 Ärzte, 186 onkologische Schwestern/ Pfleger, 386 Patienten) wurde ein Online-Fragebogen vorgelegt, der die Anwendung antiemetischer Medikation,
\end{abstract}

die Häufigkeit von Chemo-/Radiotherapieinduzierter Übelkeit und Erbrechen, deren Einfluss auf die Lebensqualität, die Compliance, Kommunikationsprobleme und demographische Faktoren abfragte.

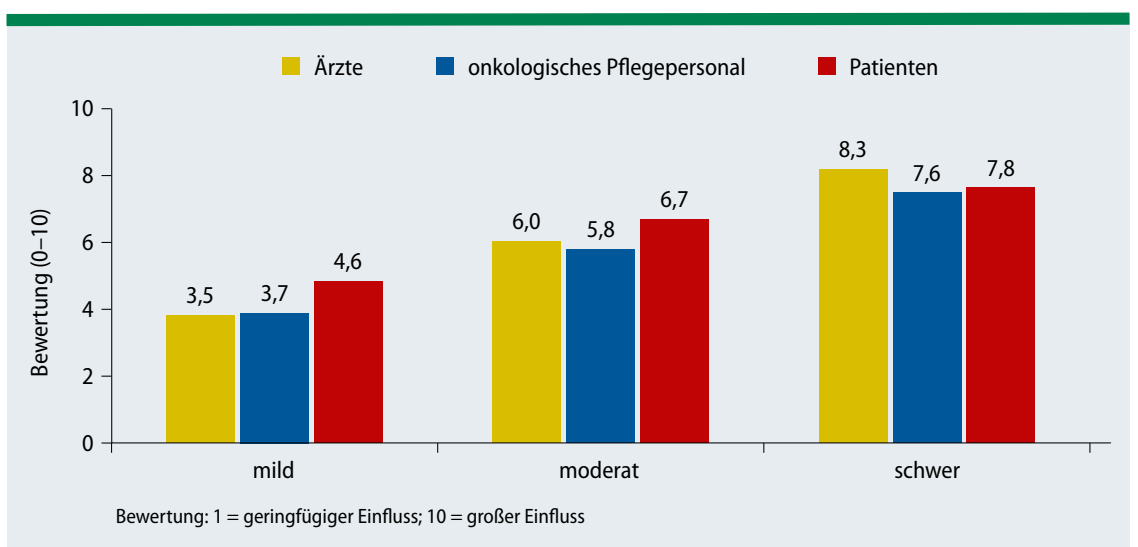

Abb. 1: Durchschnittliche Bewertung des Einflusses von Übelkeit auf das tägliche Leben.
$74 \%$ der Patienten erlebten Übelkeit, $18 \%$ Erbrechen. Ärzte und Pflegepersonal schätzten die Inzidenz von Übelkeit und Erbrechen höher ein als Patienten diese nach ihrem letzten Therapiezyklus berichteten, unterschätzten jedoch die Auswirkungen der Beschwerden auf das Alltagsleben der Patienten: Zumindest leichte und moderate Formen von Übelkeit und Erbrechen stuften sie in ihrer Bedeutung für das Alltagsleben der $\mathrm{Pa}$ tienten weniger beeinträchtigend ein, als die Patienten selbst dies erlebten (Abb. 1). $28 \%$ der Patienten empfanden, dass Onkologen die Auswirkungen der Symptome unterschätzten.

Fazit: Patienten, Ärzte und Pflegepersonal erleben Häufigkeit und Belastung von Chemo- und Radiotherapie-induzierter Übelkeit und Erbrechen unterschiedlich. Als Folge könnten antiemetische Therapien suboptimal verschrieben und angewendet werden. Kathrin von Kieseritzky

Vidall C et al. Impact and management of chemotherapy/radiotherapy-induced nausea and vomiting and the perceptual gap between oncologists/oncology nurses and patients: a cross-sectional multinational survey. Support Care Cancer. 2015;23(11):3297-305

sich die Fosaprepitant-Addition zur Standard-Antiemese der Vergleichstherapie mit alleiniger Standard-Antiemese als signifikant überlegen (78,9 vs. $68,5 \%$; $\mathrm{p}<0,001$ bzw. 77,1 vs. $66,9 \%$; $\mathrm{p}<0,001)$. In der Akutphase (0-24 h nach MECBeginn) erzielte der $\mathrm{NK}_{1}-\mathrm{RA}$ dagegen keinen Vorteil gegenüber der StandardAntiemese. Die Fosaprepitant-Gabe wurde gut vertragen.

Fazit: Auch bei Patienten mit non-AC MEC reduziert die Einmalgabe von Fosaprepitant i.v. an Tag 1 zusätzlich zur Standard-Antiemese mit einem $5-\mathrm{HT}_{3}$ RA plus Dexamethason CINV in der Spät- und in der Gesamtbeobachtungsphase.

Barbara Kreutzkamp

Weinstein C et al. Single-dose fosaprepitant for the prevention of chemotherapy-induced nausea and vomiting associated with moderatly emetogenic chemotherapy: results of a randomized, double-blind phase III trial. Ann Oncol. 2016;27(1):172-8. 